\title{
Molecular Cloning of Equine Muscle-type Phosphofructokinase cDNA
}

\author{
Tetsuo SATO ${ }^{1)}$, Takuya ITOU ${ }^{1)}$ and Takeo SAKAI ${ }^{1) *}$ \\ ${ }^{1)}$ Department of Preventive Veterinary Medicine and Animal Health, Nihon University School of Veterinary Medicine, 1866 Kameino, \\ Fujisawa, Kanagawa 252-8510, Japan
}

(Received 23 November 2002/Accepted 18 January 2003)

ABSTRACT. The complete coding region sequence of equine muscle-type phosphofructokinase $(e P F K M)$ was obtained from skeletal muscle of a thoroughbred horse. The deduced amino acid sequence of ePFKM showed $97 \%, 96 \%, 96 \%, 96 \%$ and $95 \%$ identity to canine, human, mouse, rabbit and rat PFKM, respectively. The amino and carboxyl terminal halves of ePFKM presented a structure of tandem repeat, as other mammalian PFKMs. As the amino acid residues constituting various ligand-binding sites were also conserved, it is thought that ePFKM has enzymatic activity similar to PFKM in other mammals.

KEY WORDS: cDNA, equine, phosphofructokinase.

J. Vet. Med. Sci. 65(5): 645-648, 2003

Phosphofructokinase (PFK, D-fructose 6-phosphate 1phosphotransferase, EC 2.7.1.11) uses ATP to catalyze the phosphorylation of fructose 6-phosphate to fructose 1,6-bisphosphate. This reaction is allosterically regulated by various regulators, and it is considered to be a main control point of glycolysis. There are three PFK isozymes with tissuespecific expression: muscle, liver and platelet types [3]. The cDNA sequence of the muscle type PFK (PFKM) has been determined in human [11, 13], dog [15], rabbit [8], mouse [5] and rat [10]. The evolution of $P F K$ gene based on a gene duplication from the bacterial $P F K$ has been proposed [12].

Type VII glycogenosis, due to a lack of PFKM activity, causes exertional myopathy and compensated hemolysis in humans and dogs as a rare autosomal recessive trait $[16,17]$. Multiple mutations in human PFKM, including splicing defects, frameshifts, and missense mutations, have been identified in patients from six different ethnic backgrounds establishing genetic heterogeneity of the disease [13]. Canine PFKM has been detected only a missense mutation, which changes a tryptophan codon to a stop codon [16].

The clinical behaviors of polysaccharide storage myopathy (PSSM) closely resemble type VII glycogenosis $[1,6,7$, 17]. Both disorders have PFK deficiency, and result in abnormal polysaccharide accumulation in humans and dogs. However, PFK deficiency is not evident in equines with PSSM [19]. Maximal PFK activity response to high concentration of allosteric regulators did not differ between equines with PSSM and normal equines. Therefore, equine PFKM (ePFKM) could have specific characters as difference ligand-binding sites from other mammals. Type VII glycogenosis has not been reported in equines yet. However, there is little information about $e P F K M$, only partial sequence has been described [2], and the $e P F K M$ gene has not been completely sequenced. In this study, we isolated and characterized the complete coding sequence of $e P F K M$

\footnotetext{
* Correspondence to: Prof. Sakai, T., Department of Preventive Veterinary Medicine and Animal Health, Nihon University School of Veterinary Medicine, 1866 Kameino, Fujisawa, Kanagawa 252-8510, Japan.
}

in order to obtain fundamental findings for understanding of molecular regulation in equine myopathy.

After the adult thoroughbred horse (Equus caballus) was killed by the ethical procedure at the Utsunomiya City Meat Inspection Office, Utsunomiya, Tochigi-ken, Japan, the skeletal muscle was obtained from cervix. Skeletal muscle was put into RNAlater RNA Stabilization Reagent (QIAGEN, Germany), and stored at $-20^{\circ} \mathrm{C}$.

The skeletal muscle of a thoroughbred horse was homogenized in TRIzol (GIBCO BRL, U.S.A.), and then total RNA was extracted according to the manufacturer's instructions. First strand cDNA was transcribed with $\mathrm{H}$ Minus MMuLV Reverse Transcriptase (RT, MBI Fermentas, Lithuania) and an oligo $(\mathrm{dT})_{17}$ primer. To amplify partial $e P F K M$ cDNA, several primers were designed based on published human [14], canine [15], rabbit [8] and rat [10] PFKM sequences (Table 1). Polymerase chain reactions (PCR) were performed on a PTC-0200 DNA Engine Peltier Thermal Cycler (MJ Research, U.S.A.). Forty-five cycles of amplification, including denaturation $\left(94^{\circ} \mathrm{C}, 1 \mathrm{~min}\right)$, annealing $\left(55\right.$ to $\left.62^{\circ} \mathrm{C}, 1 \mathrm{~min}\right)$, and extension $\left(74^{\circ} \mathrm{C}, 50\right.$ to $\left.60 \mathrm{sec}\right)$, were performed in HotStarTaq DNA polymerase buffer (QIAGEN) containing $1.8 \mathrm{mM} \mathrm{MgCl}_{2}, 0.2 \mu \mathrm{M}$ of each primer, $200 \mu \mathrm{M}$ dNTP, and $2.5 \mathrm{U} /$ reaction of HotStarTaq DNA Polymerase (QIAGEN). The PCR products were electrophoresed in a $1.5 \%$ agarose gel and visualized with ethidium bromide. To purify the PCR products, the amplified fragments were extracted from the agarose gel by a Gel Extraction Kit (QIAGEN).

The purified cDNA fragments were subcloned into pMOSBlue vector (Amersham Pharmacia Biotech, England) using blunt-ended cloning according to the manufacturer's instructions. Putative clones were then screened using PCR with the above primers and rTaq polymerase (TOYOBO, Japan). Thirty-five cycles of PCR were performed under the conditions described above. The nucleotide sequence of $e P F K M$ was determined using a Thermo sequence fluorescent-labeled primer cycle sequencing kit with 7-deaza-dGTP (Amersham Pharmacia Biotech) and 
Table 1. Sequences of the primers used to amplify the coding region of $e P F K M$

\begin{tabular}{|c|c|c|c|c|}
\hline Primer & Sense & Sequence & Position & PCR product \\
\hline ePFKM1S & + & 5'-GGAGAGCTAARACTAMAAGAGTGGATC-3' & -27 to & $824 \mathrm{bp}$ \\
\hline ePFKM1A & - & 5'-GCACCCTCRGCMACAATGAT-3' & +797 & \\
\hline ePFKM $2 S$ & + & 5 -GAGACAAGGACCCGTGGTTC-3, & +745 to & $804 \mathrm{bp}$ \\
\hline ePFKM2A & - & 5'-ACTGCTTCCTGCCCTCCATCA-3' & +1549 & \\
\hline e-PFKM-'- & + & 5'-GGGGGCTTTGAGGCTTACAC-3, & +1495 to & $905 \mathrm{bp}-$ \\
\hline ePFKM3A & - & 5'-GAGAGCTGACCATGATCAG-3' & +2419 & \\
\hline
\end{tabular}

Position +1 indicates the start codon, ATG. $(\mathrm{R}=\mathrm{A}$ or $\mathrm{G} ; \mathrm{M}=\mathrm{A}$ or $\mathrm{C})$

DSQ-2000L sequencer (Shimadzu, Japan) according to the manufacturer's instructions. The nucleotide sequence was finally determined by confirming that at least five clones had exhibited the identical result. DNA sequences were then analyzed using the GENETYX-WIN ver. 5.0.0 program (Genetyx, Japan) in combination with the GenBank database to compare the deduced ePFKM amino acid sequences with those from other mammalian species: human (accession No. M26066), dog (accession No. U25183), rat (accession No. U25651), mouse (accession No. AF249894) and rabbit [8].

The nucleotide sequence of $e P F K M$ reveals a 2,340 bp open reading frame that encodes a hypothetical protein of 780 amino acids with a molecular weight of $85,281 \mathrm{Da}$. $e P F K M$ cDNA has the same length of open reading frame as human, mouse, rabbit and rat PFKMs, but the canine PFKM open reading frame is two codons longer than the other mammalian PFKMs. ePFKM nucleotide and deduced amino acid sequences were deposited in the GenBank database (accession No. AB088683). Comparison of the ePFKM sequence with canine, human, mouse, rabbit and rat PFKM sequences revealed 93, 93, 90, 89 and 89\% identity, respectively, for the nucleotide sequence in the open reading frame, and 97, 96, 96, 96 and 95\% identity for the deduced amino acid sequence. Thus, $e P F K M$ has high identity with all these PFKMs. The deduced amino acid sequences of mammalian PFKMs are divided into amino (1 to 403 amino acids) and carboxyl terminal halves (404 to 780 amino acids), and aligned for maximal homology with Bacillus stearothermophilus PFK (accession No. M15643, Fig. 1) according to Eto et al. [4]. There is similarity between amino and carboxyl terminal halves of the ePFKM amino acid sequence. The primary structure of ePFKM presents gene dupulication that is tandem repeats, as observed in other mammalian PFKMs [12].

We compared the amino acid residues constituting binding sites for the substrates and regulators among the various mammalian PFKMs and with Bacillus stearothermophilus PFK. Each half of PFKM has two separate substrate-binding sites, one for ATP and the other for fructose 6-phosphate. Each half of PFKM also contains separate allosteric sites for activation by fructose bisphosphate and AMP/ADP, and sites for inhibition by citrate and ATP $[9,12]$. All residues thought to participate in ligand binding at these substrate and allosteric sites were conserved among ePFKM, other mammalian PFKMs and bacterial PFK. Furthermore, it was observed that the putative ligand-binding sites exist in the same position within the amino-terminal and carboxylterminal halves. This suggests that ePFKM enzymatic activity is similar to that of other mammalian PFKMs.

In this study, we demonstrated that various ligand-binding sites on ePFKM were conserved in other mammalian PFKM completely. This fact suggests that ePFKM acts as allosteric enzyme with a similar profile of action to canine and human PFKM. Nevertheless, the activity of PFK in equines with PSSM and normal equines did not differ in response to any allosteric regulators, in contrast to PSSMaffected humans and dogs, which have abnormalities in glycogenolytic or glycolytic pathway. Therefore, the activation of ePFKM might be controlled by special regulatory mechanism. The analysis of the transcriptional expression of $e P F K M$ gene is desirable to elucidate the metabolic control of PFK and the manifestation of PSSM in equines. Our findings in the sequencing of the $e P F K M$ gene will assist in analyzing the regulation of glycolysis by PFKM in equines. To clarify the regulation of $e P F K M$ expression, studies of 5' - and 3'-untranslated regions, intron and upstream promoter region sequences are now in progress.

\section{REFERENCES}

1. Agamanolis, D. P., Askari, A. D., DiMauro, S., Hays, A., Kumar, K., Lipton, M. and Raynor, A. 1980. Muscle Nerve. 3: 456-467.

2. Caetano, A. R., Shiue, Y. L., Lyons, L. A., O'Brien, S. J., Laughlin, T. F., Bowling, A. T. and Murray, J. D. 1999. Genome Res. 9: 1239-1249.

3. Davidson, M., Collins, M., Byrne, J. and Vora, S. 1983. Biochem. J. 214: 703-710.

4. Eto, K., Sakura, H., Yasuda, K., Hayakawa, T., Kawasaki, E., Moriuchi, R., Nagataki, S., Yazaki, Y. and Kadowaki, T. 1994. Biochem. Biophys. Res. Commun. 198: 990-998.

5. Gunasekera, D. and Kemp, R.G. 2000. Gene 260: 103-112.

6. Harvey, J.W., Calderwood, M. B., Gropp, K. E. and Denaro, F. J. 1990. Vet. Pathol. 27: 1-8.

7. Hays, A. P., Hallet, M., Delfs, J., Morris, J., Sotrel, A., Shevchuk, M. M. and DiMauro, S. 1981. Neurology 31: 1077-1086.

8. Lee, C. P., Kao, M. C., French, B. A., Putney, S. D. and Chang, S. H. 1987. J. Biol. Chem. 262: 4195-4199.

9. Li, Y., Valaitis, A. P., Latshaw, S. P., Kwiatkowska, D., Tripathi, R. L., Campbell, M. C. and Kemp, R. G. 1994. J. Biol. Chem. 269: 5781-5787. 


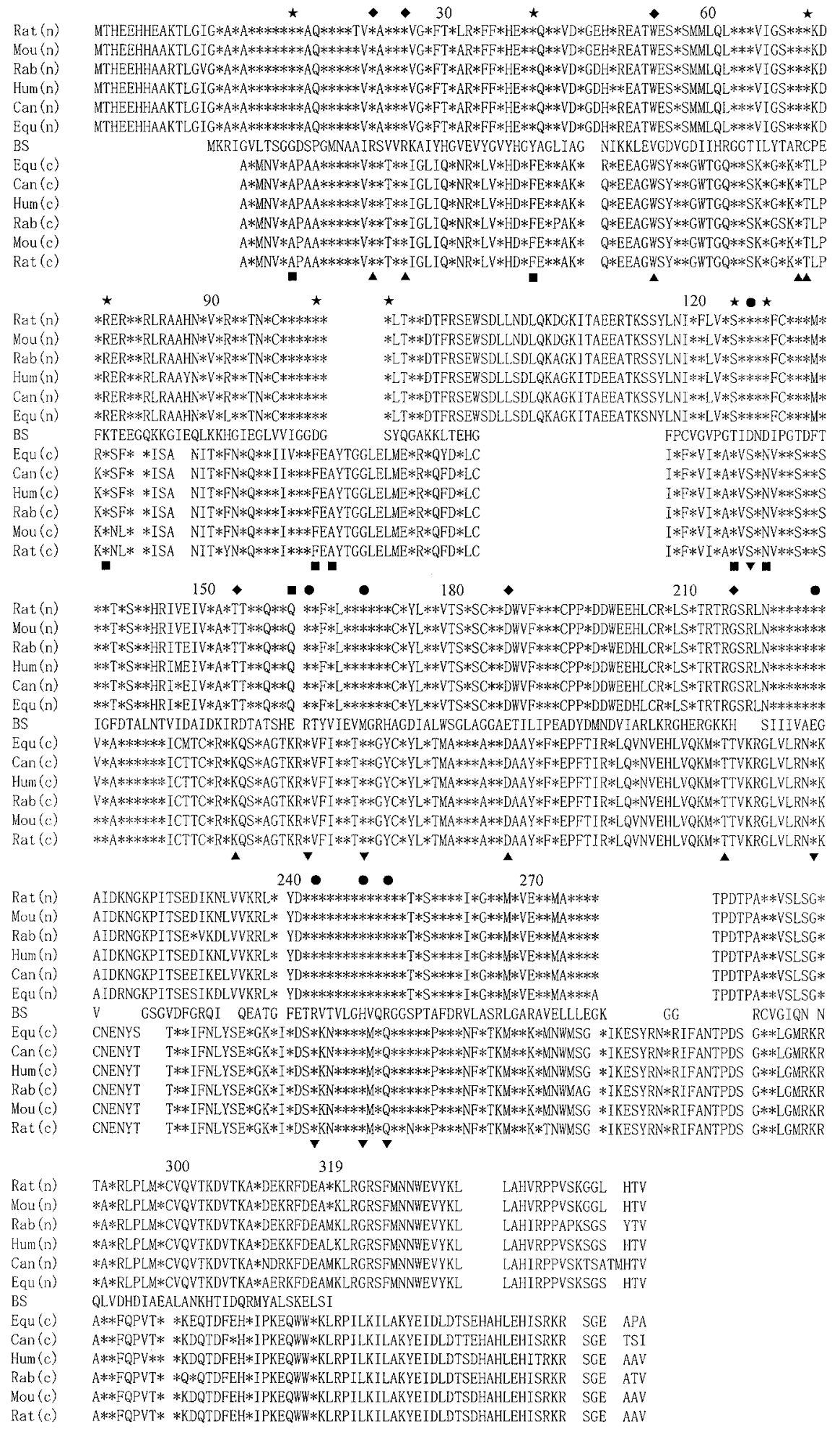

Fig. 1. Sequence comparison of equine (Equ), canine (Can), human (Hum), rabbit (Rab), mouse (Mou), rat (Rat) muscle-type and Bacillus stearothermophilus (BS) PFK. The mammalian PFKMs are divided into amino (N) and carboxyl (C) halves and aligned for maximal homology with BS PFK. Amino acid residues are numbered based on the BS PFK sequence, and asterisks indicate residues identical to those of BS PFK. Putative residues constituting various ligand-binding sites are shown by: $\star$, ATP substrate site; 0 , fructose 6-bisphosphate substrate site; ADP/AMP activation site; $\mathbf{\square}$, ATP inhibition site; $\boldsymbol{\Lambda}$, citrate inhibition site; $\boldsymbol{\nabla}$, fructose bisphosphate allosteric site. 
10. Ma, Z., Ramanadham, S., Kempe, K., Hu, Z., Ladenson, J. and Turk, J. 1996. Biochem. Biophys. Acta 1308: 151-163.

11. Nakajima, H., Noguchi, T., Yamasaki, T., Kono, N., Tanaka, T. and Tarui, S. 1987. FEBS Lett. 223: 113-116.

12. Poorman, R. A., Randolph, A., Kemp, R. G. and Heinrikson, R.L. 1984. Nature (Lond.) 309: 467-469.

13. Raben, N. and Sherman, J.B. 1995. Hum. Mutat. 6: 1-6.

14. Sharma, P. M., Reddy, G. R., Vora, S., Babior, B. M. and Mclachlan, A. 1989. Gene 77: 177-183.

15. Smith, B. F., Henthorn, P. S., Rajpurohit, Y., Stedman, H., Wolfe, J. H., Patterson, D. F. and Giger, U. 1996. Gene 168: 275-276.

16. Smith, B. F., Stedman, H., Rajpurohit, Y., Henthorn, P. S.,
Wolfe, J. H., Patterson, D. F. and Giger, U. 1996. J. Biol. Chem. 271: 20070-20074.

17. Tarui, S., Okuno, G., Ikura, Y., Tanaka, Y., Suda, M. and Nishikawa, M. 1965. Biochem. Biophys. Res. Commun. 19: $517-$ 523.

18. Vaisanen, P. A., Reddy, G. R., Sharma, P. M., Kohani, R., Johnson, J. L., Raney, A. K. and Babior, B. M. 1992. DNA Cell Biol. 11: 461-470.

19. Valberg, S. J., Townsend, D. and Mickelson, J. R. 1998. Am. J. Vet. Res. 59: 782-785.

20. Yamasaki, T., Nakajima, H., Kono, N., Hotta, K., Yamada, K., Imai, E., Kuwajima, M., Noguchi, T., Tanaka, T. and Tarui, S. 1991. Gene 104: 277-282. 